\title{
Dynamic modelling of overprinted impermeable fault gouges and surrounding damage zones as lower dimensional interfaces ${ }^{\star, \star \star}$
}

\author{
Thomas Poulet ${ }^{a, b, *}$, Martin Lesueur ${ }^{c}$ and Ulrich Kelka ${ }^{d}$ \\ ${ }^{a}$ CSIRO Mineral Resources, 26 Dick Perry Avenue, Kensington, WA 6151, Australia \\ ${ }^{b}$ School of Minerals and Energy Resources Engineering, UNSW Sydney, NSW 2052, Australia \\ ${ }^{c}$ Civil and Environmental Engineering, Duke, Durham, NC 27708-0287, USA \\ ${ }^{d}$ CSIRO Deep Earth Imaging Future Science Platform, 26 Dick Perry Avenue, Kensington, WA 6151, Australia
}

\section{ARTICLE INFO}

Keywords:

flow in fractured porous media

dynamic mixed permeability

lower dimensional interface

fault overprinting

\begin{abstract}
A B S T R A C T
In the modelling of subsurface fluid flow, faults are dominant features since they can act as fluid pathways or barriers. Special emphasis is therefore placed in representing them in a numerically efficient manner and the use of lower dimensional domains has become prevalent to simulate higher permeability features like fractures. Such features, however, only represent some of the components of natural fault networks, which can also include rather impermeable fault gouges surrounded by higher permeability damage zones for instance. Here we present a numerical approach to simulate such systems at a large scale, where the thickness of those features makes it advantageous to represent them as discrete rather than continuous domains, using lower dimensional interfaces in a conforming mesh. Benchmarks show excellent agreement with equivalent continuous simulations, regardless of the fault thickness or permeability, both for flow conduits and barriers. This approach can also account for the overprinting of faults with different permeabilities, as well as their dynamic evolution, which we illustrate with an example of trap charging. This work demonstrates the applicability of the approach to simulate fluid flow in faulted environments of various permeabilities and we discuss how those results can easily be extended to account for multi-physical processes.
\end{abstract}

\section{Introduction}

Fault networks are critical geological features that can control the fluid flow pattern in the upper crust above the brittle-ductile transition (Rawling, Goodwin and Wilson, 2001; Wibberley, Yielding and Toro, 2008; Faulkner, Jackson, Lunn, Schlische, Shipton, Wibberley and Withjack, 2010). As such, assessing the permeability of these structure and being able to accurately simulate flow and transport in these networks is of considerable interest in a number of fields including mineral exploration (Barnicoat, Sheldon and Ord, 2009) oil and gas production (Ferronato, Gambolati, Janna and Teatini, 2008), natural gas storage (Gasanzade, Bauer and Pfeiffer, 2019), $\mathrm{CO}_{2}$-storage (Tillner, Langer, Kempka and Kühn, 2016), or radioactive waste disposal (Tsang, Neretnieks and Tsang, 2015).

Due to complex hydraulic, mechanical and chemical interactions that occur in fault zones, these structures exhibit a wide range of permeability values, and can form flow conduits or barriers for instance. These scenarios represent two end members and combined barrier-conduit structures are likely to form in low-permeability strata (Bense and Person, 2006; Faulkner et al., 2010; Bauer, Schröckenfuchs and Decker, 2016). Field observations have also shown that porosity and permeability distributions in fault systems can be time-dependent, as active faults can experience short episodic periods of fluid flow triggered by seismic activity, followed by post-seismic healing (Louis, Luijendijk, Dunkl and Person, 2019). These dynamic permeability changes occur both ways, with increases also due to extensional fractures (Cappa, 2009) and decreases resulting from post-seismic healing (Aben, Doan, Gratier and Renard, 2017) or clay smear (Vrolijk, Urai and Kettermann, 2016). Those processes can be temporary, permanent, or episodic (Cox, 2016; Alevizos, Poulet and Veveakis, 2014), with opening and closing of porosities occurring at various time scales. Additionally, the sole consideration of different time scales can lead to a single feature forming both a barrier at human

${ }^{\star}$ T.P. lead drafting the manuscript and developing the methodology. M.L. developed the interface implementation. U.K. provided the geological expertise. All authors contributed to the writing of the manuscript.

${ }^{\star \star}$ Declarations of interest: None.

*Corresponding author

@ thomas.poulet@csiro.au (T. Poulet)

ORCID(s): 0000-0001-7351-3083 (T. Poulet); 0000-0001-9535-2268 (M. Lesueur) 
time scales and a pathway at geological times (Wibberley et al., 2008). This ambivalent behaviour clearly highlights the importance of identifying and deepen our understanding of the fluid flow dynamics in fault networks.

Accurate flow and transport simulations in fault networks rely on appropriate discretization of the fault zone architecture, where the system should only be simplified as an end member (conduit or barrier) for problems where those assumptions are perfectly justified. In this study we focus on common geological scenarios which include a mix of permeability features, with a basic conceptual model comprising a low-permeability fault core (gouge) surrounded by a more permeable damage zone (Caine, Evans and Forster, 1996; Barnicoat et al., 2009; Cappa and Rutqvist, 2011; Faulkner et al., 2010; Sutherland, Toy, Townend, Cox, Eccles, Faulkner, Prior, Norris, Mariani, Boulton, Carpenter, Menzies, Little, Hasting, Pascale, Langridge, Scott, Lindroos, Fleming and Kopf, 2012; Yamashita and Tsutsumi, 2017). This particular - yet quite wide-spread - case makes it unlikely to capture accurately regional fluid flow dynamics by representing faults zones simply as conduits or barriers. Based on field observations in the Dixon Valley, Caine et al. (1996) have estimated the permeability contrasts between host-rock, damage zone and fault core to be two to three orders of magnitude. In such a case the faults act as transversal flow barriers but as longitudinal fluid conduits and these individual structural elements need to be distinguished conceptually and treated accordingly when modelling such scenarios.

In a subsurface system, fault cores represent geometrically thin features and the computational costs for resolving such features numerically can be very high. This results both from the considerable number of fine mesh elements required as well as the associated time-step size reduction to accommodate smaller elements. This fact led to the development of various methods to model Discrete Fracture Networks (DFN), where the permeability of the host rock matrix is negligible compared to the permeability of the thin features, or Discrete Fracture Methods (DFM), compromising on the homogenisation simplification to still account for some of the matrix properties like its permeability for instance. Efficient methods have been introduced to simulate thin features in a discrete manner (see review from Berre, Doster and Keilegavlen, 2018), with their thickness or aperture not considered geometrically in the mesh, providing great numerical efficiency over the continuum approach where all features are considered and meshed explicitly. Such techniques cover the whole spectrum of permeabilities, with some handling both the cases of fluid conduits and barrier (e.g. Martin, Jaffré and Roberts, 2005; Angot, Boyer and Hubert, 2009). Even the pre-processing step of meshing the fracture or fault network has been greatly simplified with the development of powerful meshing packages (e.g. Geuzaine and Remacle, 2009) for conforming methods accounting for all features in the mesh, as well as nonconforming methods (e.g. Schädle, Zulian, Vogler, Bhopalam, Nestola, Ebigbo, Krause and Saar, 2019) which can integrate those features without meshing them within the matrix.

A convenient concept to simulate thin features consists in considering them as lower-dimensional manifolds (Alboin, Jaffré, Roberts and Serres, 2002). This approach is gaining in popularity (see review from Berre et al., 2018) and provides an efficient way to model highly permeable features, which is the most common end-member considered when fractures are involved. From this perspective, considering other cases becomes a question of introducing extra relevant constraints (Schädle et al., 2019), like imposing non-standard Robin type conditions at the interface to handle flow barriers for instance (Martin et al., 2005).

In this contribution, we present an approach to simulate the fluid flow in barrier-conduit systems which comprise faults of spatially and temporally varying permeabilities. Our proposed method accounts for the anisotropy associated with fault zone architecture, distinguishing between longitudinal flow pathways (e.g. in the surrounding damage zone) and transversal barriers across the fault core.

\section{Method}

Various methods exist to simulate conforming Discrete Fracture Matrix (DFM) models on conforming meshes, as shown in the review from Berre et al. (2018). In this section, we start with a popular approach, showing some of its strengths and weaknesses, before introducing additional degrees of freedom to palliate those deficiencies and better simulate our specific problem or interest. Three discrete approaches are presented and compared to a reference continuum case. The schematic of all methods is shown in Fig. 1 in 2D for clarity.

Among the numerous numerical platforms available, we chose to use the open-source REDBACK simulator (Poulet, Paesold and Veveakis, 2017) to implement all features presented in this study, based on the Multiphysics Object Oriented Simulation Environment (MOOSE ) (Gaston, Newman, Hansen and Lebrun-Grandió, 2009). REDBACK was specifically designed to investigate problems of fault reactivation (e.g. Veveakis, Alevizos and Poulet, 2017) and the flexibility of MOOSE is particularly well adapted to develop new features independently of the dimensionality of the 


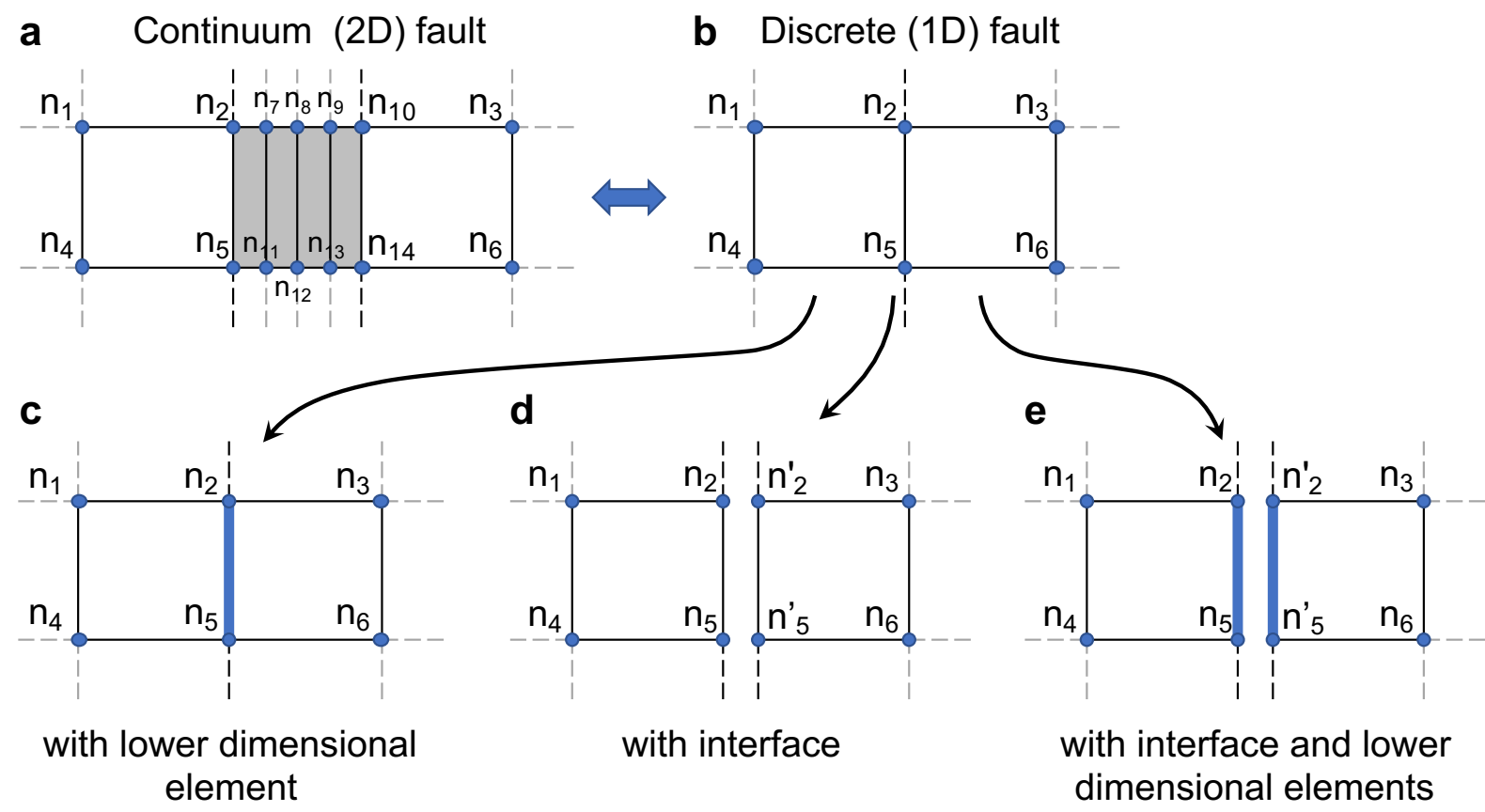

Figure 1: Schematics of the various mesh types used to model a fault, shown here in 2D between two quadrilatera elements; (a) Using 2D elements (in grey) to mesh the fault across its thickness, using an implicit continuum approach, used as reference in this study; (b) Using a discrete (1D) approach, with the fault handled at the common edge of the elements (nodes $n_{2}$ and $n_{5}$ ) in three various ways shown in the remaining subfigures; (c) 1D fault handled with additional lower-dimensional element between nodes $n_{2}$ and $n_{5}$; (d) 1D fault modelled by an interface between sets of duplicated nodes $\left(n_{2}^{\prime}\right.$ and $\left.n_{5}^{\prime}\right)$ and their superposed counterparts $\left(n_{2}\right.$ and $\left.n_{5}\right)$, shown here in exploded view (slightly shifted) for visualisation purposes; and (e) 1D fault modelled with both (i) an interface between the edge nodes $\left(n_{2}\right.$ and $\left.n_{5}\right)$ and their superposed duplicated images $\left(n_{2}^{\prime}\right.$ and $\left.n_{5}^{\prime}\right)$ in exploded view and (ii) lower-dimensional elements on the corresponding edges $\left(n_{2}-n_{5}\right.$ and $\left.n_{2}^{\prime}-n_{5}^{\prime}\right)$.

meshes used and also to modify, where necessary, the meshes themselves, which were created in this study using GMSH (Geuzaine and Remacle, 2009).

\subsection{Lower dimensional elements}

A popular DFM method is using co-dimension one domains (Berre et al., 2018), to represent thin geological features like faults or fractures as infinitely thin geometries, while accounting for their actual thickness and behaviour through the system of equations solved. MOOSE provides this functionality to use lower dimensional domains inside the overall model (Schädle, Wilkins, Ebigbo and Saar), and this has been used for the purpose of modelling flow in fractured media (Cacace and Jacquey, 2017; Schädle et al., 2019). This method, shown in Fig. 1, removes an important level of complexity at the meshing stage and it is also numerically more efficient than the corresponding continuum method (Fig. 1a). Indeed, avoiding elements of very small size to discretise the thickness of the fault not only reduces the total number of elements but also increases accordingly the timestep size that can be used in the simulation. An additional set of co-dimension one elements are added as a new subdomain to the mesh to connect the existing nodes on the fault ( $n_{2}$ and $n_{5}$ on Fig. 1c), superposed to the face of existing elements. Any system of equations can be solved on such subdomains, which provides an easy and powerful way to run for example complex multi-physics simulations (e.g. Cacace and Jacquey, 2017). For the purpose of comparing methods, we restrict ourselves to the simplest case of hydraulic diffusion, which can simply be described in dimensionless form as

$$
\partial_{t} p=D \partial_{i} \partial_{i} p
$$

where $P$ denotes the dimensionless pore pressure, $t$ the dimensionless time and $D$ the diffusivity. 

scaled by the same factor $a$.

\subsection{Interface} middle region $R_{3}$ on Fig. 2 .

$$
D^{(R)} p_{, i}^{(R)} n_{i}^{(F)}=D^{(F)} p_{, i}^{(F)} n_{i}^{(F)},
$$
$(A)$ and $(B)$, is computed as

$$
p_{, i}^{(F)} n_{i}^{(F)}=\frac{\Delta p}{a}=\frac{p^{(A)}-p^{(B)}}{a} .
$$

$$
\begin{aligned}
& D^{(A)} p_{, i}^{(A)} n_{i}^{(F)}-D^{(F)} \frac{p^{(B)}-p^{(A)}}{a}=0, \\
& D^{(B)} p_{, i}^{(B)} n_{i}^{(F)}-D^{(F)} \frac{p^{(B)}-p^{(A)}}{a}=0 .
\end{aligned}
$$
for diffusion at the interface.

To account for the mixed dimensionality of the various subdomains in the variational formulation, all terms integrated on the lower dimensional elements need to be multiplied by the thickness of the fault or aperture of the fracture, $a$, as described in (Schädle et al.). For the diffusion term, the same effect can be obtained by using a diffusivity value

This method allows accurate modelling of highly permeable faults with longitudinal flow, but it is not designed to model transversal flow, since the lower dimensional elements are infinitely thin and therefore "invisible" transversally. This issue has been addressed (e.g. Martin et al., 2005) to model flow barriers as well as fractures, and this can be achieved in MOOSE by enriching the problem with an additional constraint on the transversal flow, creating interfaces on the faults to account for the transition between both sides of each fault.

Simulating transversal flow across an infinitely thin feature requires extra constraints (e.g. Martin et al., 2005; Angot et al., 2009) with a discontinuous approach, where all nodes on the fault are duplicated and superposed, as shown on Fig. 1d. Note that this figure is shown in exploded view, so all pairs of duplicated nodes $\left(n_{i}\right.$ and $\left.n_{i}^{\prime}\right)$ can be distinguished visually. This approach can be easily implemented in MOOSE through its interface functionality, following (Lesueur, 2019), where the contribution to the variational form can be described between pairs of duplicated nodes, in this instance expressing the continuity of flux between the regions on each side of the interface, replacing the

The method presented in the previous paragraph focused on describing permeable faults or fractures but this represents only one half of the spectrum. Another very interesting feature of faults is their ability to seal off fluids when their permeability is very low. However, the problem of flow barriers is radically different from fluid conduits and the lower-dimensionality method is not adapted to deal with those system. Specifically, the fluid flow is controlled in this case by the transversal low diffusivity of the fault that needs to be precisely modelled. This paragraph presents an approach to solve fluid flow through a very low permeability fracture. Since an impermeable fault gouge is a relatively thin feature, the flow in the fault is considered to always remain in steady-state.

When modelled as a flat interface as intended for numerical cost, the flow barrier results in a discontinuity of pressure at the fault core. In order to handle discontinuities at the interface, we duplicate nodes at the interface, that still superpose at the same coordinate. Each node represents one side of the interface and the relationship between those two duplicated nodes is given by a prescribed interface law. In the case of hydraulic diffusion, the interface law is simply derived from the continuity of flux across each side of the interface, expressed as

where the superscripts $(F)$ and $(R)$ refer to the fault (interface) and neighbouring regions (reservoir) respectively.

The gradient of pressure between the two sides of the interface $(F)$ of effective thickness $a$, denoted by the exponents

Combining Eqs. 3 and 2 we end up with a relationship for the interface law defined as:

Implementing the interface law for diffusion in a finite element code consists in adding the second term of the equations above to the respective residuals of node (A) and node (B), as the first term represents the natural boundary conditions

To verify the implementation of this pore pressure interface, we compare the results against an analytical problem for a simple one-dimensional case. We consider three regions $\left(R_{1}, R_{2}, R_{3}\right)$ of various thicknesses $\left(D_{1}, D_{2}, D_{3}\right)$ and permeability values $\left(k_{1}, k_{2}, k_{3}\right)$, and solve for the steady-state of the $1 \mathrm{D}$ horizontal flow through as (Fig. 2). We apply 

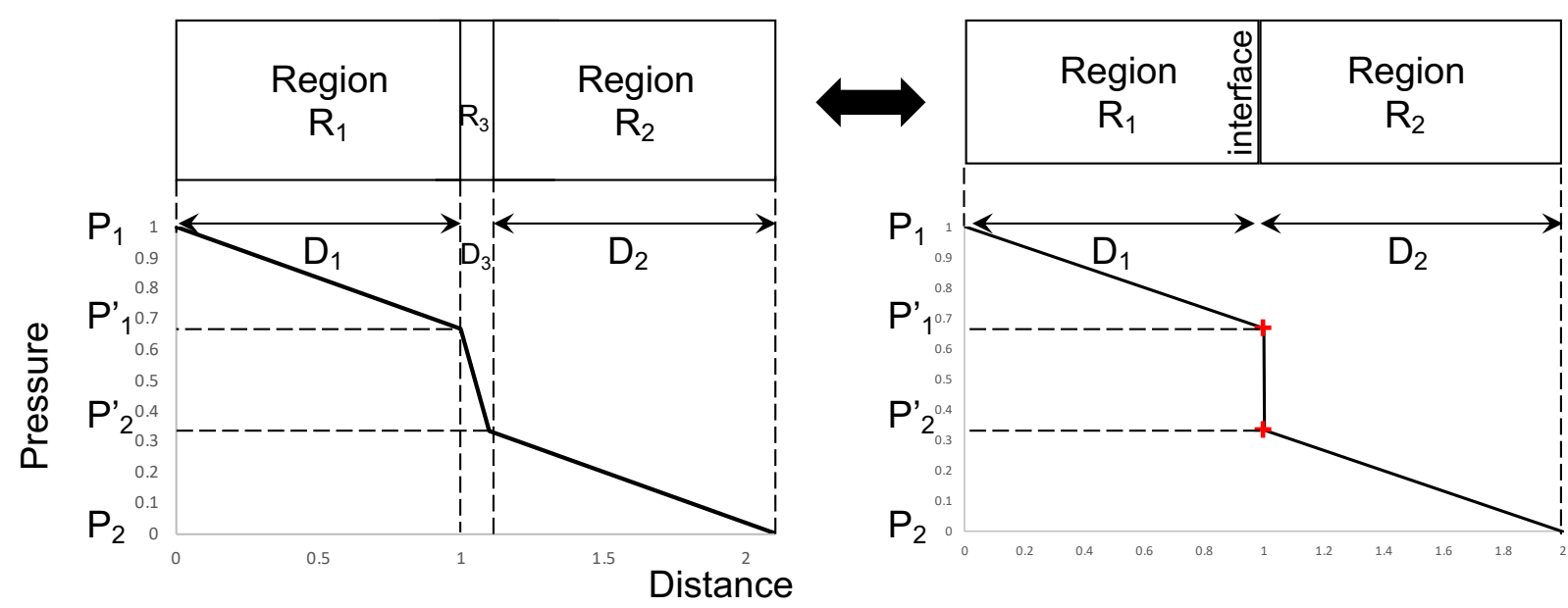

Figure 2: Model used to benchmark the flow interface. The specific case plotted is described by $D 1=D 2=10 \times D 3$; $p_{1}=1$ and $p_{2}=0 ; k_{1}=k_{2}=10 \times k_{3}$. On the left are plotted the result for the system fully resolved and on the right for the reduced system with interface. The system with interface matches with machine precision the results of the full system, highlighted with the red crosses.

a pressure gradient across these three blocks with constant boundary conditions at each end $\left(p_{1}\right.$ and $\left.p_{2}\right)$. The two pressures $p_{1}^{\prime}$ and $p_{2}^{\prime}$ at the interface between the regions 1-3 and 2-3 can be expressed as:

$$
\begin{array}{r}
p_{2}^{\prime}=\frac{k_{1} k_{3} D_{2} D_{3} p_{1}+k_{2} D_{3}\left(k_{1} D_{3}+k_{3} D_{1}\right) p_{3}}{\left(k_{3} D_{2}+k_{2} D_{3}\right) *\left(k_{1} D_{3}+k_{3} D_{1}\right)-k_{3} k_{3} D_{1} D_{2}}, \\
p_{1}^{\prime}=\frac{k_{1} D_{3} p_{1}+k_{3} D_{1} p_{2}^{\prime}}{k_{1} D_{3}+k_{3} D_{1}} .
\end{array}
$$

This analytical solution is used to validate the numerical results obtained by solving a similar problem where the middle region is replaced by a flat interface, with the setup and results shown in Fig. 2, demonstrating perfect agreement. More detailed benchmark results are presented in Sec. 3.

We note that compared to the lower-dimensional approach, which was kernel agnostic, this method requires a specific translation to an interface law for each physical process considered. Still, adding more complexity is not complicated and we demonstrate this by adding gravity effects to the interface law for Darcy's law. Eq. 5 becomes:

$$
\begin{aligned}
& -\frac{k^{(A)}}{\mu_{f}}\left(p_{, i}^{(A)}-\rho_{f} g_{i}\right) n_{i}^{(F)}+\frac{k^{(F)}}{\mu_{f}}\left(\frac{p^{(B)}-p^{(A)}}{a}-\rho_{f} g_{i} n_{i}^{(F)}\right)=0, \\
& -\frac{k^{(B)}}{\mu_{f}}\left(p_{, i}^{(B)}-\rho_{f} g_{i}\right) n_{i}^{(F)}+\frac{k^{(F)}}{\mu_{f}}\left(\frac{p^{(B)}-p^{(A)}}{a}-\rho_{f} g_{i} n_{i}^{(F)}\right)=0 .
\end{aligned}
$$

\subsection{Combining lower dimensional elements and interface}

With the lower dimensional subdomains of Sec. 2.1 capturing longitudinal flow and the interface of Sec. 2.2 handling transversal flow barriers, we now combine both approaches to simulate any combination of fault permeabilities. In particular, we target the case of sealing fault cores bordered by permeable damage zones on either side by using the approach shown schematically in Fig. 1e, with an interface between duplicated set of nodes along the fault, along with one extra lower dimensional subdomain on either side of the fault. This particular setup allows to simulate the longitudinal flow independently on either side of a barrier, with the sum of the scaling factors for both subdomains (see Sec. 2.1) adding up to the thickness $a$ of the fault. Without any specific information, both are taken as $a / 2$. This approach is tested and compared to the previous two in a series of benchmarks presented in Sec. 3.2. 

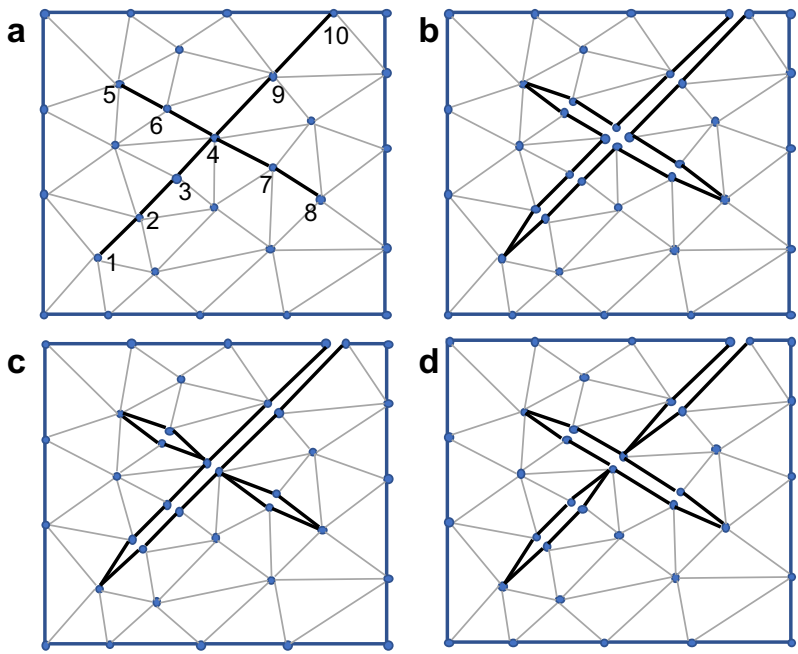

Figure 3: Schematic of node splitting for handling fault connections and intersections; (a) initial mesh, with two intersecting sets of edges (involving 10 nodes) marked as faults; (b) corresponding mesh topology after node splitting, with some nodes duplicated (and superposed, but shown shifted for visualisation purposes) once (nodes 2, 3, 6, 7, 9, 10) or three times (node 4); nodes $(1,5,8)$ at the extremities of the faults but not on the boundaries are not duplicated; (c) and (d) both possible cases of fault overprinting with extremity node (4) duplicated only once.

\subsection{Mesh generation}

To use this approach, some interfaces with duplicated nodes need to be added to the original mesh, along with a pair of lower dimensional subdomains on either side of those interfaces, paying particular attention to the cases of faults intersections. While this step represents conceptually a pre-processing step independent from the simulation itself, we implemented in REDBACK a functionality as a MeshGenerator to generate such a mesh from an original one where faults are simply tagged as sidesets. This function can handle the cases of faults cross-cutting the domain boundaries and intersecting each other, either fully or partially. Those cases are conceptually displayed in Fig. 3. Fig. 3a shows an example of a 2D mesh with two faults intersecting each other, one of which touches the top boundary. Fig. 3b shows the equivalent exploded view, with all nodes on the original faults duplicated, except at fault extremities not on the boundary (nodes $1,5 \& 8$ ). In this example, the node (index 4) at the intersection of both faults ends up duplicated three times. Fig. 3b\&c show how to handle cases of fault overprinting. The intersecting node is only duplicated for the latest geological fault, allowing longitudinal flow through the intersection along that fault. The other (earlier) one needs to be defined in two parts, one on each side.

\section{Results \& discussion}

In this section we showcase the capabilities of the approach presented in Sec. 2, to model faults of various permeabilities - flow channels and barriers - as lower dimensional interfaces, through several benchmarks and applications.

\subsection{Generic example}

To begin with, we illustrate the approach by solving the transient pressure diffusion problem (eq. 1) on a 3D block of size 1 containing three discrete faults of thickness 0.001, shown in Fig. 4a. The faults are five times more permeable than the matrix along their surfaces (see Sec. 2.1), but with a transversal permeability (across their thickness) which is ten times lower than the matrix. Starting from a uniform zero pressure in the model, a constant dimensionless pressure value of 1 is imposed at the bottom of the model and Fig. 4b shows the typical patterns obtained from fracture flow through highly permeable features. Fig. 4c, however, which displays pressure profiles on the faults only, highlights the effect of the transversal permeability, with the pressure diffusing faster around the fault than across them. To better understand the impact of the longitudinal and transversal permeabilities, some quantitative benchmarks are required. 


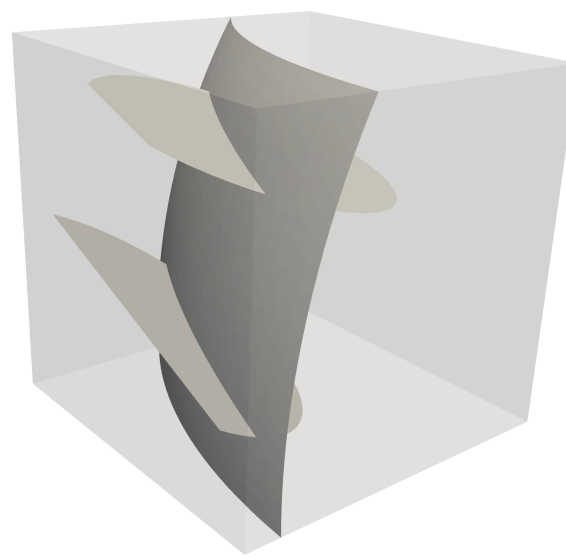

(a)

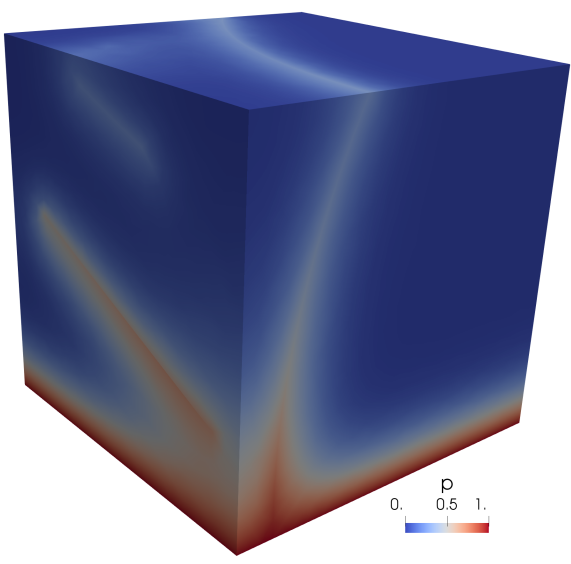

(b)

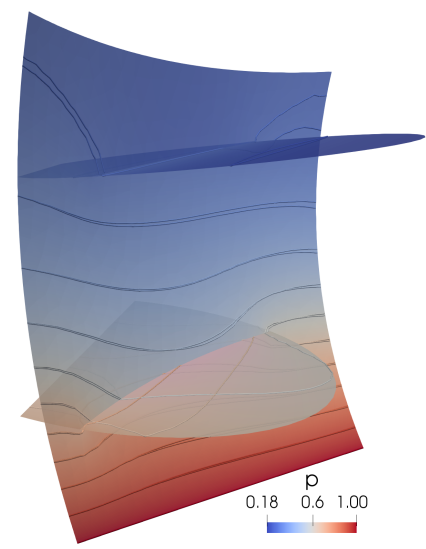

(c)

Figure 4: Generic scenario of pressure diffusion in a faulted block. (a) Geometry, two oval faults intersecting a curved fault (as the implementation is not restricted to linear features). (b) Pressure profile highlighting the high longitudinal permeability of the faults. (c) Pressure contours on the faults (rotated view), highlighting the lower transversal permeability of the faults. Note the apparent duplication and shift of the isobaric lines, which emphasises the differential of pressure between the two faces of the fault.
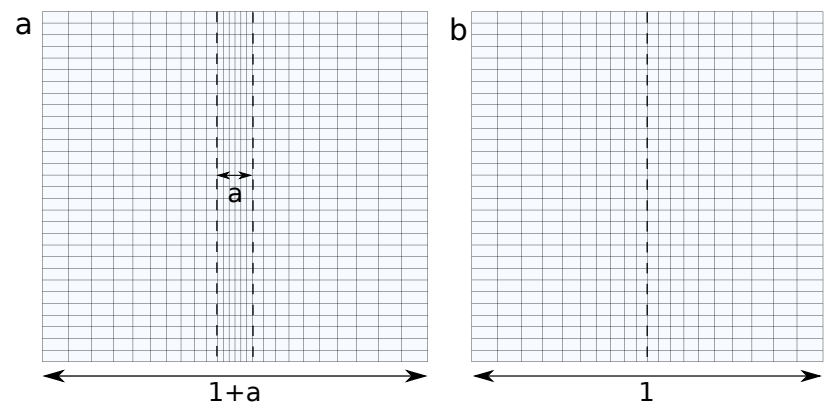

Figure 5: Benchmark meshes used for the (a) continuum approach, with a vertical fault of aperture $a$ meshed in the centre, and (b) discrete approach, with a vertical fault indicated by a dashed line at the interface between two columns of mesh elements.

\subsection{Benchmarks}

Various benchmarks have been proposed to assess fluid flow implementations through thin features, faults or fractures (e.g. Shao, He, Hokr, Gardner, Kunz and Balvin, 2016; Hokr, Shao, Gardner, Balvín, Kunz, Wang and Vencl, 2016; Cacace and Jacquey, 2017), yet they most often focus distinctly either on conduits or barriers, but not necessary on a mix of both. We propose therefore to test our approach on a simple scenario of hydraulic diffusion through a $2 \mathrm{D}$ rectangular domain containing a single feature, to compare the effects of the various implementations on the longitudinal and transversal flow components.

\subsubsection{Setup}

We solve the problem of dimensionless pressure diffusion (eq. 1) on a 2D unitary square, for clarity, containing a single vertical feature in its centre (Fig. 5). This geometry is meshed in two different manners, using the continuum and discrete approaches presented in Sec. 2. With the continuum approach, shown in Fig. 5a, the feature of thickness $a$ is represented explicitly with its true thickness and meshed as the other regions of the domain, leading to a total width of the domain of dimension $1+a$. On the other hand, the discrete approach, shown in Fig. 5b, only represents the thin feature implicitly (shown in dashes on the figure) in between the two central columns of elements, keeping a total width of 1 . The matrix surrounding the central feature is meshed identically for both types of meshes, with each half of the domain represented by $10 \times 30$ elements (see Fig. 5). For the continuum approach, the central feature is meshed 
with six elements across.

In all cases, a constant initial pressure is prescribed over the whole domain $\left(P_{\text {init }}=0\right)$ and two sets of boundary conditions (BC) are used to compare the longitudinal and transversal flows, by setting a constant over-pressure $(P=1)$ either on the top or on the left boundary. Three cases are investigated, for a diffusivity within the central feature $\left(D_{f}\right)$ which is ten times larger, equal to, or ten times lower than the diffusivity of the surrounding matrix $\left(D_{m}\right)$. The resulting effects are then compared on two different meshes, for fault thicknesses $a=0.01$ and $a=0.1$. The following three subsections present the results of the methods described in Sec. 2.1, 2.2 and 2.3 on the 12 respective cases (2 boundary conditions $\times 2$ fault thicknesses $\times 3$ diffusivity contrasts), all compared with the reference results obtained with the continuum approach, in which the fault thickness is explicitly accounted for in the mesh.

In order to test the implementation of the time dependency, all benchmarks are performed on a transient simulation, for an arbitrary total dimensionless time $t_{\text {end }}=5$ with a time step $d t=0.1$. To evaluate the results, the pressure profiles are plotted on a horizontal cross-section at mid-height, i.e. on a profile perpendicular to the fault at $y=0.5$. The results obtained with discrete approaches are superposed across the matrix on top of the reference results computed with the continuum approach, leaving a gap across the fault where the pressure is not resolved.

\subsubsection{Lower dimensional elements}

First, we simulate the flow diffusion in the discrete approach using only lower dimensional elements, following the method described in Sec. 2.1 and the results are shown in Fig. 6. As expected, all subfigures for longitudinal flow (top BC, white backgrounds) show perfect matches between the discrete and continuum results, regardless of the fault thickness (which is irrelevant in that case) or diffusivity contrast between the fault and the matrix. The subfigures for transversal flow (left BC, grey backgrounds), however, demonstrate the limitations of this method, which is only valid for transversal flow across highly diffusive faults but does not capture the effect of a fault with equal or lower permeability than the matrix, with the discrepancy dramatically increasing as the fault thickness increases (e.g. Fig. 6j).

\subsubsection{Interface}

Next, we run the same set of scenarios using the discrete method presented in Sec. 2.2, which was designed to account for flow barriers. Fig. 7 shows the comparison of the results against the respective solutions obtained with the continuum approach. These results show that the method captures indeed most of the effects of flow barriers for transversal flow (left BC, grey backgrounds), with a discrepancy remaining due to the transient effects not being appropriately accounted for, that becomes more obvious with increasing fault thickness. The method does not capture at all any effect of longitudinal flow (top BC, white backgrounds) if the fault diffusivity differs from the matrix one.

\subsubsection{Combined Interface and lower dimensional elements}

Finally, we test the same scenarios using a combination of the two previous methods, as described in Sec. 2.3. Fig. 8 shows perfect agreement for every single case, regardless of the fault thickness, diffusivity contrast between the fault and matrix, or direction of the flow. This approach captures very well the longitudinal flow thanks to the lower dimensional elements, but also the transversal flow thanks to the interface. Note that the appropriate handling of the transience from the lower dimensional elements also eliminates the small discrepancy observed for transversal flow in Sec. 3.2.3.

\subsection{Mixed permeability contrasts}

After the validation of the approach on a simple benchmark of Sec. 3.2, we now look at an example to illustrate an application involving two intersecting faults of different permeabilities, respectively 100 times higher and lower than the value the permeability for the matrix background. We solve the flow on a unit square with Dirichlet boundary conditions for the dimensionless pressure on the left-hand side $(P=1)$ and right-hand side $(P=0)$ of the model. The sealing fault crosses the whole height of the model and is intersected by the more transmissive one, as shown on Fig. 9. Following Sec. 2.3, the overprinting of one fault by the other is important to consider and both scenarios are therefore presented.

Fig. 10 shows the results obtained using both the discrete approach of Sec. 2.3 (Fig. 10c\&d) and a continuum approach, for reference, in which the faults are meshed across their thickness (Fig. 10a\&b). Both meshes have a relatively fine mesh, with a similar number of triangles $(\approx 24,000$ for the discrete one and $\approx 25,000$ for the continuum one). Fig. $10 \mathrm{a} \& \mathrm{c}$ show the results for the case where the sealing fault overprints the other and forms a complete flow barrier, while Fig. 10b\&d represent the other case where the permeable fault provides a channel piercing through the 


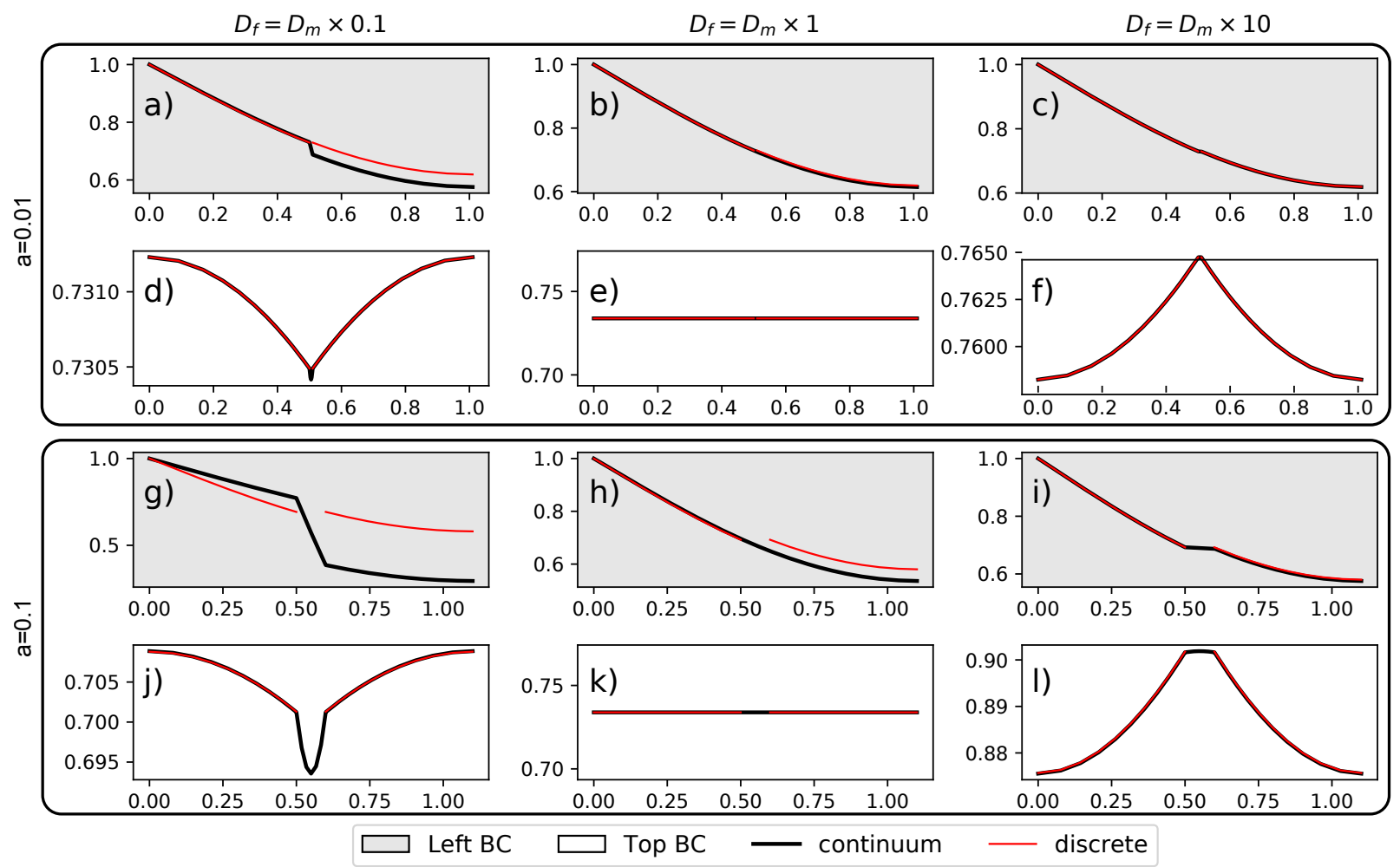

Figure 6: Benchmark results comparing the continuum (black line) and discrete (red line) cases for the lower dimensional element implementation on two different geometries, with a fault thickness of $a=0.01$ (a-f) and $a=0.1$ (g-l), as described on Fig. 5. The three columns show various cases of fault diffusivity $\left(D_{f}\right)$, respectively smaller than, equal to, and larger than the matrix diffusivity $\left(D_{m}\right)$. All profiles show the pressure (y-axis) on a horizontal cross-section (x-axis) at mid-height of the model. Two sets of fixed pressure boundary conditions were used to force a flow from the left (grey background) or from the top (white background). Results show good agreement for the case of top flow, but the fault remains invisible to any transversal flow (from the left, white backgrounds).

barrier. The results are in perfect agreement, as illustrated by the identical patterns of the isobars between the top and bottom rows of Fig. 10, showing only the difference of thickness of the fault where the permeable feature ends, towards the upper centre of the model.

\subsection{Trap charging}

Finally, we demonstrate an illustrative scenario of fault reactivation for a 3D reservoir within an antiform, in the shape of a dome, intersected by and displaced along a $60^{\circ}$ dipping fault, inside a unit cube bounding box, which showcases the ability to model dynamic permeability changes. The permeability of the reservoir is taken as reference with a normalised diffusivity of $D_{\text {res }}=1$, corresponding to a permeability value of $0.1 \mathrm{D}$. The rest of the volume is mostly taken as more impermeable, to be representative of a cap rock, with a diffusivity of $10^{-4}(0.01 \mathrm{mD})$, except for the fault which is modelled using the approach described in Sec. 2.3. The transversal diffusivity of the fault is considered to be $10^{-6}(0.1 \mu \mathrm{D})$, representing a barrier, while the longitudinal diffusivity of the fault on either side is taken as $10^{4}(1,000 \mathrm{D})$, representing some more permeable damaged zones very close to the fault. Note that all values are presented in dimensionless forms as the purpose of this study is to highlight the capabilities of the method rather than simulate a realistic geological scenario. The pressure is initialised with a 0 value at the top of the model and a hydrostatic gradient.

In this scenario, we simulate qualitatively the two important phases of a reservoir forming in a trap and discharging due to the fault reactivation (Wibberley et al., 2008), accounting for Darcy flow and gravity (Eqs. $8 \& 9$ for the interface law). The trap charging involves a pressure build-up within the lower part of the reservoir, taking place at geological time scales. The very short fault reactivation event, on the other hand, sees an abrupt increase of the transversal 


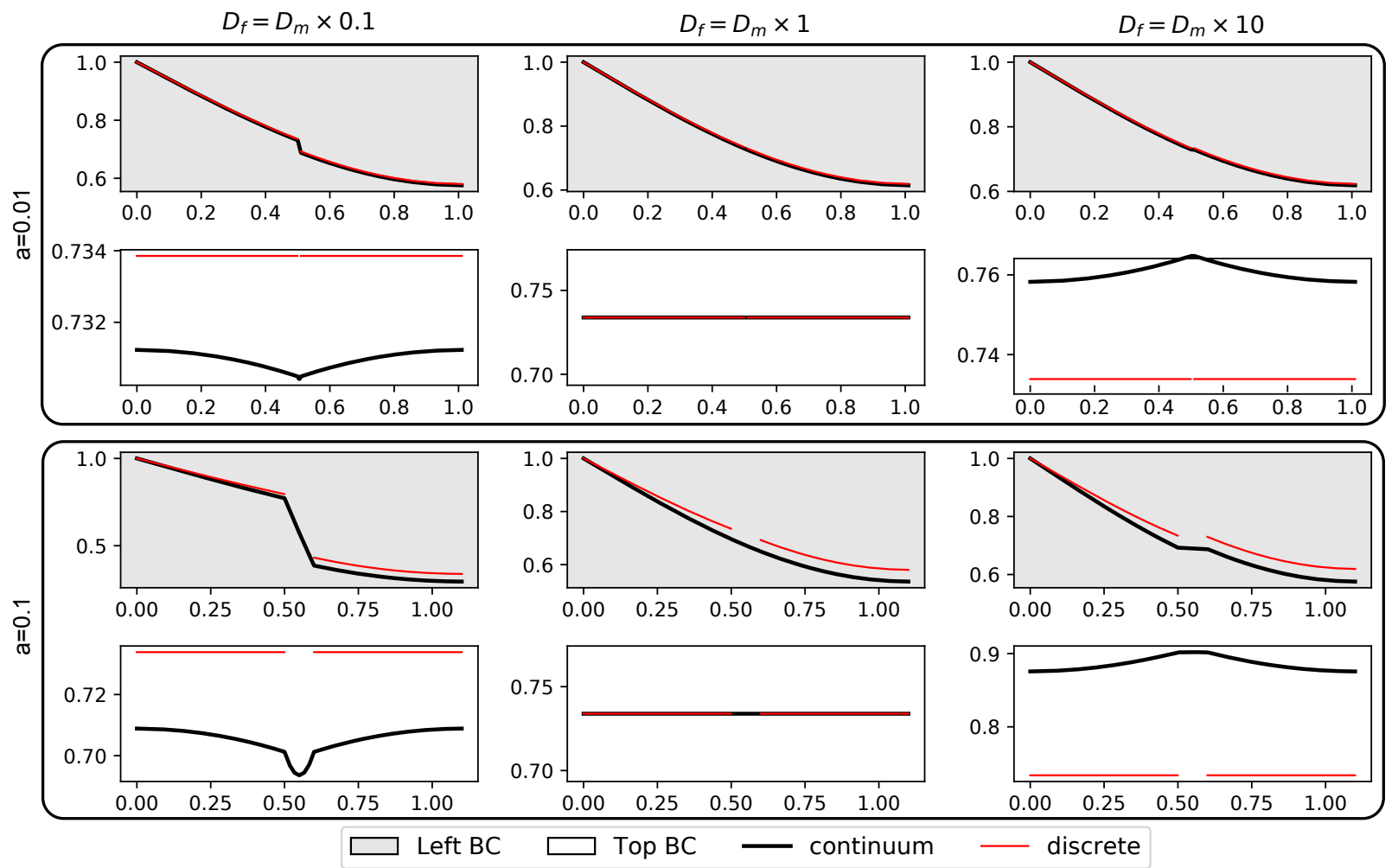

Figure 7: Benchmark profiles for interface implementation showing appropriate handling for horizontal flow (left BC, grey background), increasingly better as the fault gets thinner (smaller $a$ ). The longitudinal diffusivity, however, is not accounted for and the fault remains invisible from the top (top BC, white backgrounds). the next trap. A Neumann boundary condition - constant flux - for the dimensionless pressure is applied on left face of the bottom part of the reservoir for the whole duration of the simulation, from $t=-1,200,00$ a to $t=600,000 \mathrm{a}$. At $t=0$, the transversal diffusivity of the fault is artificially increased by four orders of magnitude (Nguyen, Guglielmi, Graupner and Rutqvist, 2019) to $1 \mathrm{mD}$, in order to simulate a seal failure during a fault reactivation event.

Fig. 11 and 12 show three snapshots of the simulation results for the pressure and fluid flow, at various times during the simulation. Fig. 11a shows the pressure distribution in the model after the first timestep (when the flow just started charging the lower part of the reservoir). Fig. $11 \mathrm{~b}$ shows the pressure just before the fault reactivation (at $t=0$ ), highlighting the pressure build-up in the lower part of the reservoir, with fluid flowing along the fault, but barely entering the upper part of the reservoir, mostly - but not completely - sealed by the fault. Fig. 11c at the end of the simulation, when the upper part of the reservoir has already been charged considerably. Fig. 12a and Fig. 12c show the corresponding fluid flow velocities at the beginning and end of the simulation, where only the input flow boundary condition is visible, while Fig. $12 \mathrm{~b}$ shows the flux slightly after reactivation, at $t=8,000 \mathrm{a}$, when the overpressure from the closest part of the lower reservoir to the fault is triggering some equilibration flow to the upper part of the reservoir near the (now transmissive) fault. Fig. 13 shows the pore pressure evolution at two sampling points on either side of the fault, in the upper part of the model, with the same coordinates (since the fault is infinitely thin) highlighted by the small grey point on Fig. 11. Fig. 13a shows the results during the whole simulation and Fig. 13b zooms in around the reactivation event. Those results show clearly the trap charging in the lower part of the reservoir over long time scales, as well as the effects of the abrupt fault reactivation, leading to a large transversal permeability increase of the fault, followed by the consequent charging of the upper part of the reservoir. The pressure is naturally building up much more rapidly in the zones connected by high permeability until reactivation, with Fig. 13a highlighting the pressure differential building across the fault in the upper part of the reservoir, before both sides equilibrate during a short time dictated by the fault thickness and its increased permeability. The pressure equilibration of Fig. 13b correspond to the flow distribution of Fig. 12b. Note that before the reactivation $(t<0)$ the pressure in the upper reservoir along the 

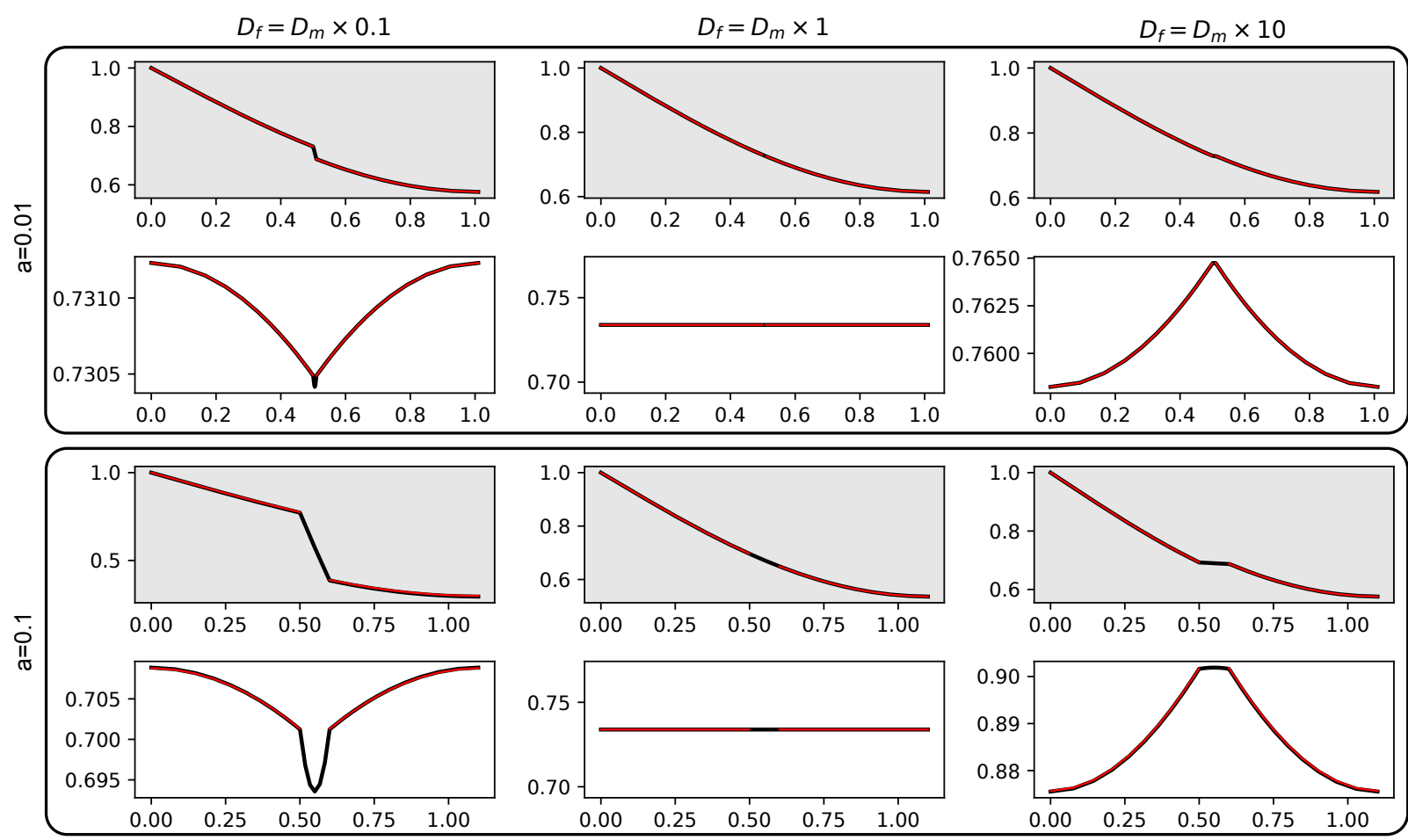

$\square$ Left BC

$\square$ Top BC - continuum discrete

Figure 8: Benchmark profiles for implementation with both interface and lower dimensional blocks. This implementation combines the best of both approaches and matches all cases, even improving on the interface handling for the thicker fault $(a=0.1)$.

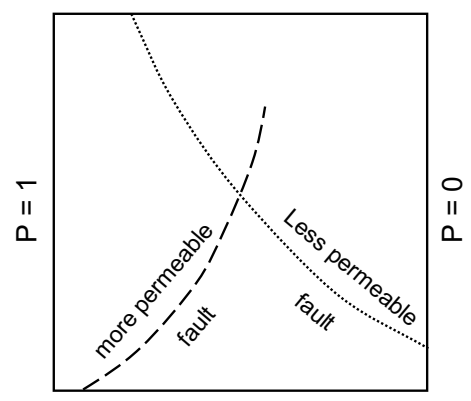

Figure 9: Setup of intersection faults with mixed permeability contrasts, showing the unitary square model, location of the faults and boundary conditions. time-scale considered (Wibberley et al., 2008).

\section{Conclusion}

In this contribution we presented an accurate method to model impermeable faults bordered by more permeable damage zones at a larger scale where the overall thickness of those features becomes small enough not to be meshed as a continuum, hence reducing the computational cost. The method combines the advantages of using lower dimensional domains, to capture the longitudinal flow in the higher permeability zones, with an interface between duplicated mesh nodes on the fault to handle the pressure discontinuity. This allows for simultaneously simulating barriers and 


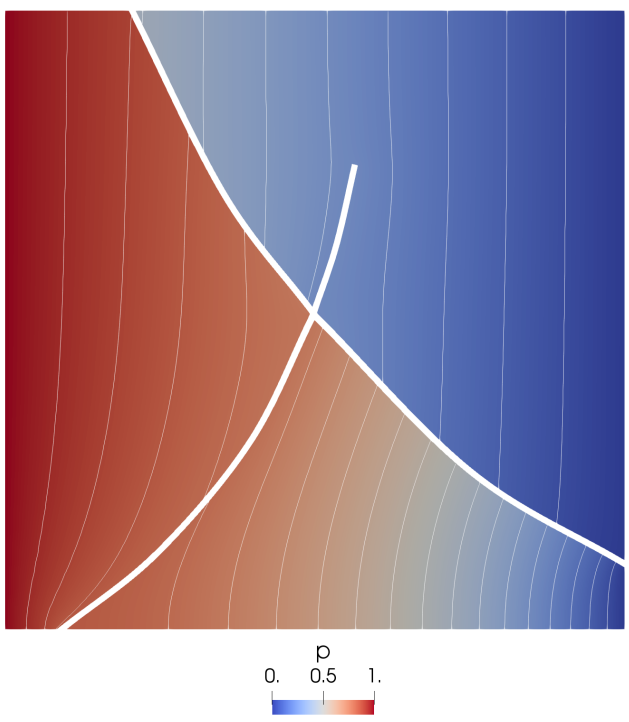

(a)

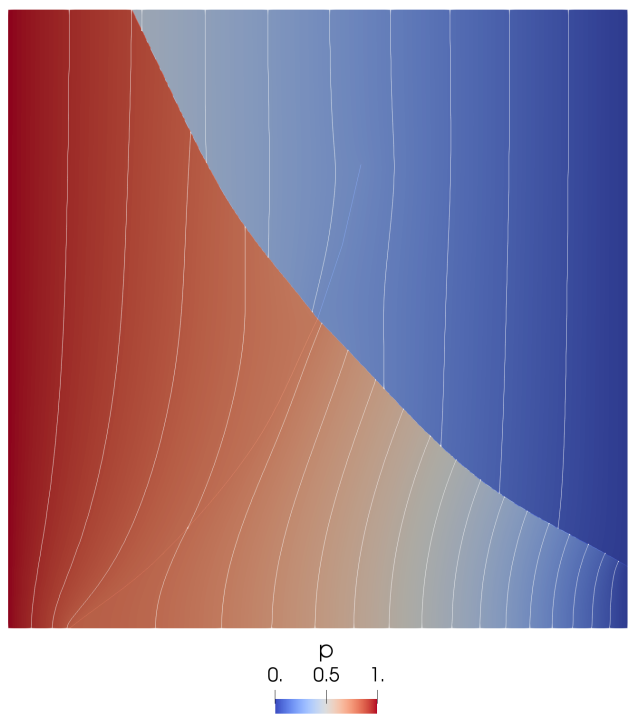

(c)

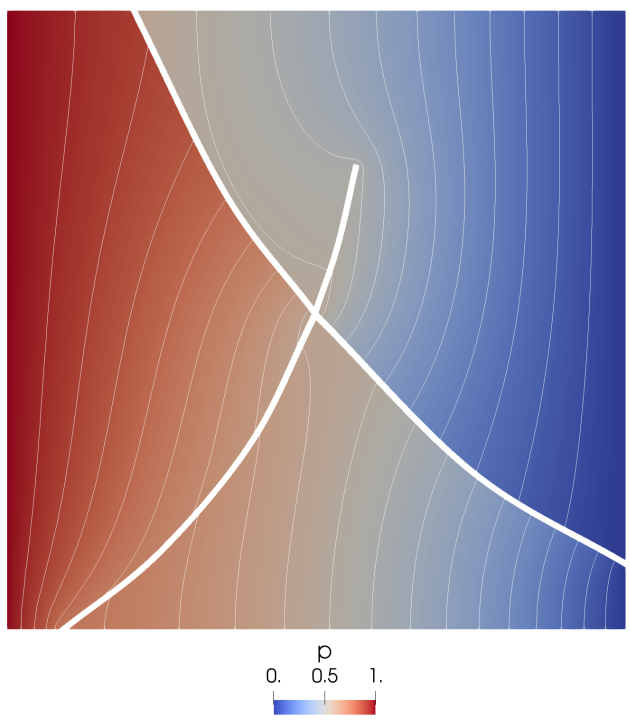

(b)

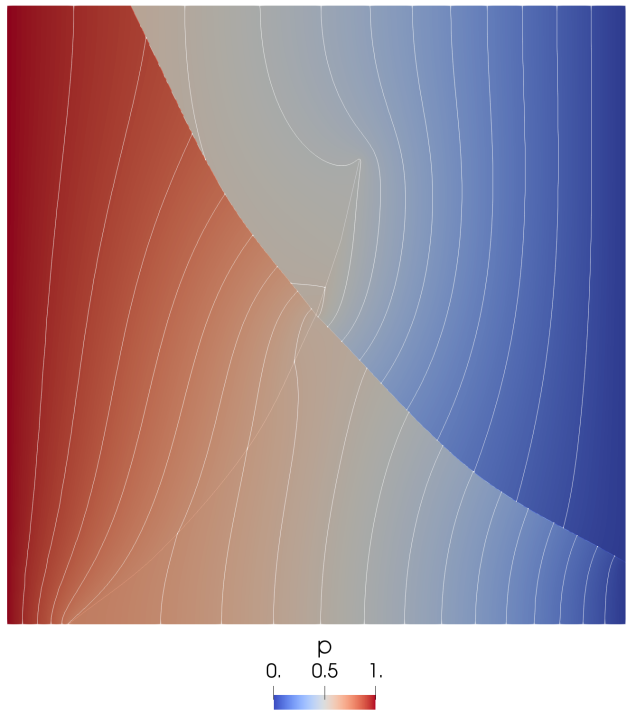

(d)

Figure 10: Pressure $(p)$ distributions for two scenarios involving intersecting faults, one with higher and one with lower permeability than the matrix, following Fig. 9, modelled with a continuum (a \& b) or discrete (c \& d) approach. The first column (a \& c) shows the case where the lower permeability fault (downwards from left to right) overprints the higher permeability fault (upwards from left to right). The second column ( $b$ \& d) shows results for the case where the higher permeability fault overprints the lower permeability one.

highly permeable faults, or fractures, and therefore accounting for all structural elements of fault zones. Benchmarks (Sec. 3.2) showed that our discrete approach provides an accurate representation of the transient (and steady state) hydraulic problem on faults of any geometry. Note that the interface method is limited to solving steady-state transversal flow but in combination with low-dimensional domains allows for solving transient problems as well. Our approach is also applicable to capturing dynamic permeability evolutions, for instance during fault reactivation (see example in Sec. 3.4). Finally, we showed how the method can be applied to account for overprinting faults, by treating the intersections appropriately in the pre-processing step. It is interesting to note that the lower dimensional method is completely generic as it uses a compelling feature of MOOSE where the system equations is independent of the mesh. 


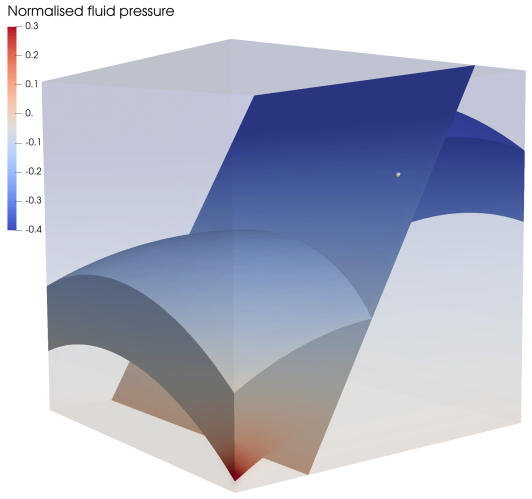

(a) At first simulation timestep

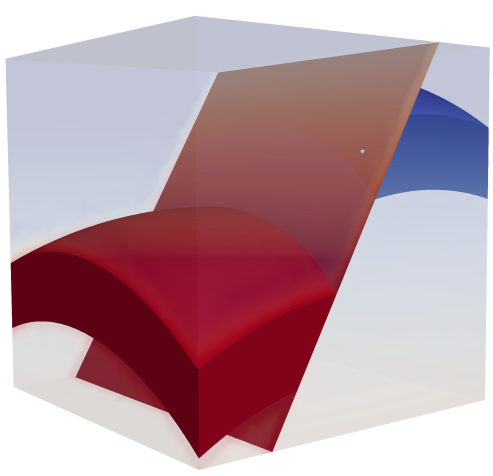

(b) Just after fault reactivation

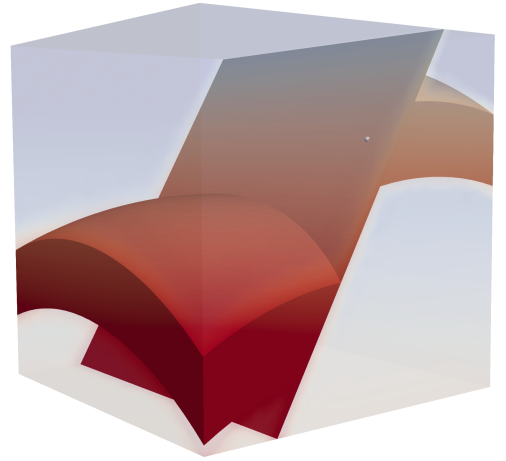

(c) At simulation end

Figure 11: Normalised fluid pressure evolution during reservoir simulation. The pressure builds up first in the lower reservoir, propagates along the fault, and only builds in the upper reservoir after fault reactivation increases the transversal permeability of the fault.

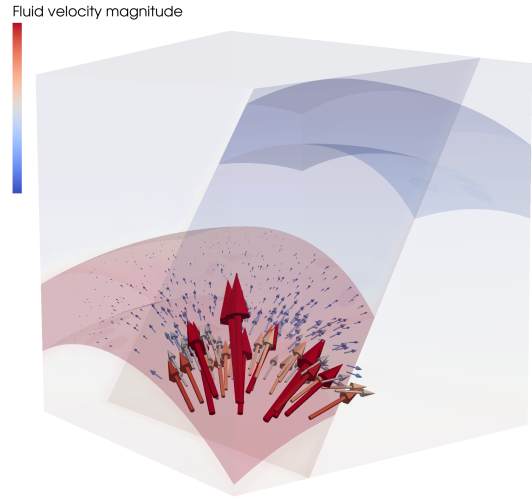

(a) just before fault reactivation

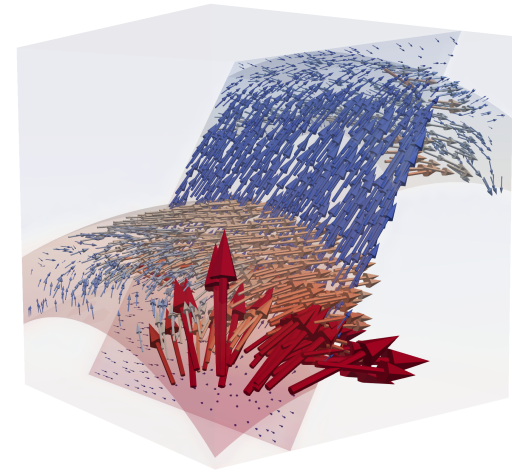

(b) Fluid flow shortly after fault reactivation

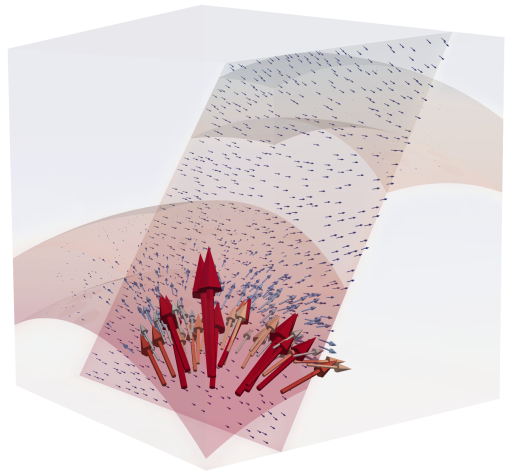

(c) Fluid flow at simulation end

Figure 12: Fluid flow evolution corresponding to scenario shown in Fig. 11. Note that the magnitude of flow arrows is exaggerated ten times in the fault for visualisation purposes (for all three subfigures) and the flow around the source inflow is only shown after a short distance to mask the otherwise overprinting arrows.

As such, all existing MOOSE implementations of any physical processes can run natively on lower dimensional domains. The interface method, however, requires a specific implementation of the physical processes considered across the discontinuity. We showed here the common case of hydraulic flow with gravity and more physical processes will be added following the same methodology. The simple case of diffusion can already capture scenarios including thermal and hydraulic processes. This paves the way to detailed large scale simulations in various fields including the study of ore deposits, reservoir simulation in faulted environments, fault reactivation, carbon capture and storage, or nuclear waste disposal to name only a few.

\section{Computer code availability}

All results presented in this study were obtained with the open-source REDBACK simulator (Poulet et al., 2017) (http://github.com/pou036/redback), a MOOSE module (Gaston et al., 2009). 

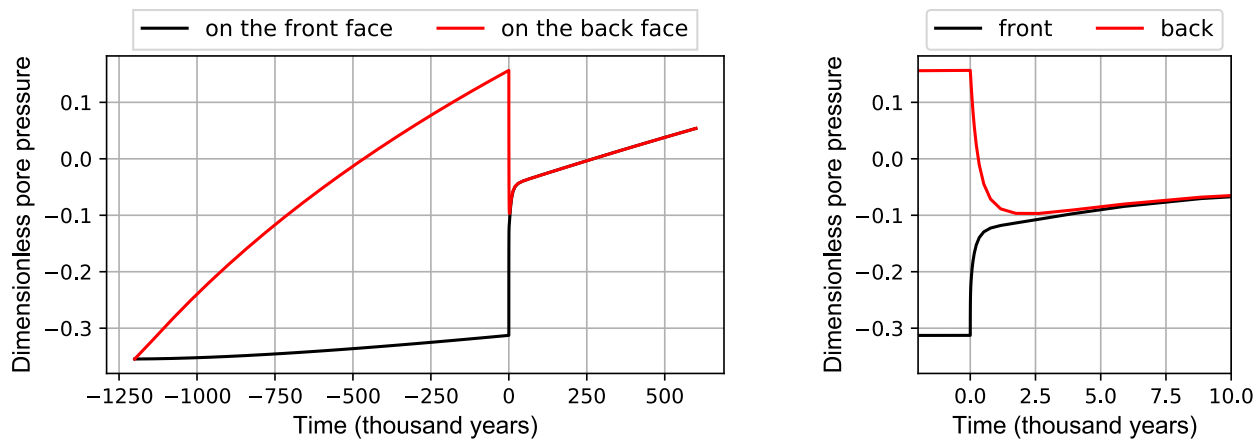

Figure 13: Time evolution of normalised pore pressure at location marked by a grey sphere on Fig. 11, on either sides of the fault, with fault reactivation at $t=0$; (a) full simulation; (b) zoom on the first 10,000 years after reactivation

\section{References}

Aben, F.M., Doan, M.L., Gratier, J.P., Renard, F., 2017. Experimental postseismic recovery of fractured rocks assisted by calcite sealing. Geophysical Research Letters 44, 7228-7238. doi:10.1002/2017GL073965.

Alboin, C., Jaffré, J., Roberts, J.E., Serres, C., 2002. Modeling fractures as interfaces for flow and transport in porous media, in: Chen, Z., Ewing, R.E. (Eds.), Fluid Flow and Transport in Porous Media: Mathematical and Numerical Treatment, American Mathematical Society. pp. 13-24. doi:10.1090/conm/295/04999.

Alevizos, S., Poulet, T., Veveakis, E., 2014. Thermo-poro-mechanics of chemically active creeping faults. 1: Theory and steady state considerations. Journal of Geophysical Research: Solid Earth 119, 4558-4582. doi:10.1002/2013JB010070.

Angot, P., Boyer, F., Hubert, F., 2009. Asymptotic and numerical modelling of flows in fractured porous media. ESAIM: Mathematical Modelling and Numerical Analysis 43, 239-275. doi:10.1051/m2an/2008052.

Barnicoat, A.C., Sheldon, H.A., Ord, A., 2009. Faulting and fluid flow in porous rocks and sediments: implications for mineralisation and other processes. Mineralium Deposita 44, 705-718. doi:10.1007/s00126-009-0236-4.

Bauer, H., Schröckenfuchs, T.C., Decker, K., 2016. Hydrogeological properties of fault zones in a karstified carbonate aquifer (northern calcareous alps, austria). Hydrogeology Journal 24, 1147-1170. doi:10.1007/s10040-016-1388-9.

Bense, V.F., Person, M.A., 2006. Faults as conduit-barrier systems to fluid flow in siliciclastic sedimentary aquifers. Water Resources Research 42. doi:10.1029/2005WR004480.

Berre, I., Doster, F., Keilegavlen, E., 2018. Flow in fractured porous media: A review of conceptual models and discretization approaches. Transport in Porous Media doi:10.1007/s11242-018-1171-6.

Cacace, M., Jacquey, A.B., 2017. Flexible parallel implicit modelling of coupled thermal-hydraulic-mechanical processes in fractured rocks. Solid Earth 8, 921-941. doi:10.5194/se-8-921-2017.

Caine, J.S., Evans, J.P., Forster, C.B., 1996. Fault zone architecture and permeability structure. Geology 24, 1025-1028. doi:10.1130/ 0091-7613(1996)024<1025:FZAAPS>2.3.CO;2.

Cappa, F., 2009. Modelling fluid transfer and slip in a fault zone when integrating heterogeneous hydromechanical characteristics in its internal structure. Geophysical Journal International 178, 1357-1362. doi:10.1111/j.1365-246X . 2009.04291.x.

Cappa, F., Rutqvist, J., 2011. Modeling of coupled deformation and permeability evolution during fault reactivation induced by deep underground injection of CO2. Int. J. Greenhouse Gas Control 5, 336-346. doi:10.1016/j . ijggc. 2010.08.005.

Cox, S.F., 2016. Injection-driven swarm seismicity and permeability enhancement: Implications for the dynamics of hydrothermal ore systems in high fluid-flux, overpressured faulting regimes - an invited paper. Economic Geology 111, 559-587. doi:10.2113/econgeo.111.3.559.

Faulkner, D., Jackson, C., Lunn, R., Schlische, R., Shipton, Z., Wibberley, C., Withjack, M., 2010. A review of recent developments concerning the structure, mechanics and fluid flow properties of fault zones. Journal of Structural Geology 32, 1557-1575. doi:10 . 1016/j • jsg . 2010.06 .009.

Ferronato, M., Gambolati, G., Janna, C., Teatini, P., 2008. Numerical modelling of regional faults in land subsidence prediction above gas/oil reservoirs. International Journal for Numerical and Analytical Methods in Geomechanics 32, 633-657. doi:10.1002/nag.640.

Gasanzade, F., Bauer, S., Pfeiffer, W.T., 2019. Sensitivity analysis of gas leakage through a fault zone during subsurface gas storage in porous formations. Advances in Geosciences 49, 155-164. doi:10.5194/adgeo-49-155-2019.

Gaston, D., Newman, C., Hansen, G., Lebrun-Grandió, D., 2009. Moose: A parallel computational framework for coupled systems of nonlinear equations. Nuclear Engineering and Design 239, 1768 - 1778. doi:10.1016/j.nucengdes . 2009.05.021.

Geuzaine, C., Remacle, J.F., 2009. Gmsh: A 3-d finite element mesh generator with built-in pre- and post-processing facilities. International Journal for Numerical Methods in Engineering 79, 1309-1331. doi:10.1002/nme. 2579.

Hokr, M., Shao, H., Gardner, W.P., Balvín, A., Kunz, H., Wang, Y., Vencl, M., 2016. Real-case benchmark for flow and tracer transport in the fractured rock. Environmental Earth Sciences 75. doi:10.1007/s12665-016-6061-z.

Lesueur, M., 2019. Influence of multiphysics couplings across scales: from digital rock physics to induced fault reactivation. Ph.D. thesis. UNSW Sydney. School of Minerals and Energy Resources Engineering.

Louis, S., Luijendijk, E., Dunk1, I., Person, M., 2019. Episodic fluid flow in an active fault. Geology 47, 938-942. doi:10.1130/G46254.1.

Martin, V., Jaffré, J., Roberts, J.E., 2005. Modeling fractures and barriers as interfaces for flow in porous media. SIAM Journal on Scientific Computing 26, 1667-1691. doi:10.1137/s1064827503429363. 
Nguyen, T.S., Guglielmi, Y., Graupner, B., Rutqvist, J., 2019. Mathematical modelling of fault reactivation induced by water injection. Minerals 9 , 282. doi:10.3390/min9050282.

Poulet, T., Paesold, M., Veveakis, E., 2017. Multi-physics modelling of fault mechanics using redback - a parallel open-source simulator for tightly coupled problems. Rock Mechanics and Rock Engineering 50, 733-749. doi:10.1007/s00603-016-0927-y.

Rawling, G.C., Goodwin, L.B., Wilson, J.L., 2001. Internal architecture, permeability structure, and hydrologic significance of contrasting fault-zone types. Geology 29, 43-46. doi:10.1130/0091-7613(2001) 029<0043: IAPSAH>2.0. CO; 2.

Schädle, P., Wilkins, A., Ebigbo, A., Saar, M.O., . Flow through an explicitly fractured medium and flow through a fractured network. https: //mooseframework.inl.gov/moose/modules/porous_flow/flow_through_fractured_media.html. Accessed: 2019-10-01.

Schädle, P., Zulian, P., Vogler, D., Bhopalam, S.R., Nestola, M.G., Ebigbo, A., Krause, R., Saar, M.O., 2019. 3d non-conforming mesh model for flow in fractured porous media using lagrange multipliers. Computers \& Geosciences 132, 42-55. doi:10.1016/j . cageo.2019.06.014.

Shao, H., He, W., Hokr, M., Gardner, P.W., Kunz, H., Balvin, A., 2016. Flow processes, in: Thermo-Hydro-Mechanical-Chemical Processes in Fractured Porous Media: Modelling and Benchmarking. Springer International Publishing. Terrestrial Environmental Sciences, pp. 33-39. doi: 10.1007/978-3-319-29224-3_3.

Sutherland, R., Toy, V.G., Townend, J., Cox, S.C., Eccles, J.D., Faulkner, D.R., Prior, D.J., Norris, R.J., Mariani, E., Boulton, C., Carpenter, B.M., Menzies, C.D., Little, T.A., Hasting, M., Pascale, G.P.D., Langridge, R.M., Scott, H.R., Lindroos, Z.R., Fleming, B., Kopf, A.J., 2012. Drilling reveals fluid control on architecture and rupture of the alpine fault, new zealand. Geology 40, 1143-1146. doi:10.1130/g33614.1.

Tillner, E., Langer, M., Kempka, T., Kühn, M., 2016. Fault damage zone volume and initial salinity distribution determine intensity of shallow aquifer salinisation in subsurface storage. Hydrology and Earth System Sciences 20, 1049-1067. doi:10.5194/hess-20-1049-2016.

Tsang, C.F., Neretnieks, I., Tsang, Y., 2015. Hydrologic issues associated with nuclear waste repositories. Water Resources Research 51, $6923-6972$. doi:10.1002/2015WR017641.

Veveakis, E., Alevizos, S., Poulet, T., 2017. Episodic tremor and slip (ets) as a chaotic multiphysics spring. Physics of the Earth and Planetary Interiors 264, 20-34. doi:10.1016/j.pepi.2016.10.002.

Vrolijk, P.J., Urai, J.L., Kettermann, M., 2016. Clay smear: Review of mechanisms and applications. Journal of Structural Geology 86, 95-152. doi:10.1016/j.jsg.2015.09.006.

Wibberley, C.A.J., Yielding, G., Toro, G.D., 2008. Recent advances in the understanding of fault zone internal structure: a review. Geological Society, London, Special Publications 299, 5-33. doi:10.1144/sp299.2.

Yamashita, T., Tsutsumi, A., 2017. Fluid-flow properties of fault zones, in: Involvement of Fluids in Earthquake Ruptures. Springer Japan, pp. 51-71. doi:10.1007/978-4-431-56562-8_3. 